\title{
The laboratory identification of gram-positive anaerobic cocci
}

\author{
D. A. MURDOCH and I. J. MITCHELMORE*
}

Department of Medical Microbiology, St Bartholomew's Hospital, London EC1A 7BE

\begin{abstract}
Summary. A collection of 256 clinical strains and 40 reference strains of gram-positive anaerobic cocci (GPAC) was studied, to characterise the recognised species more fully and to define groups of strains which might correspond to previously undescribed species. The methods used were: gas-liquid chromatography (GLC) for the detection of volatile fatty acids (VFAs); determination of the pre-formed enzyme profile with a commercially available kit, ATB 32A; microscopic appearance; colonial morphology; and antibiotic sensitivity tests. Strains were placed in one of five VFA groups according to their GLC profile; $96 \%$ of strains were further assigned to 12 groups by their enzyme profile. There was $>99 \%$ agreement between the two methods.

Of 111 clinical strains in the VFA-negative group, 110 gave one of three distinct enzyme profiles corresponding to Peptostreptococcus magnus, P. micros and P. heliotrinreducens. The assignment of strains to groups based on their microscopic appearance and colonial morphology agreed well with groupings according to enzyme profile. Identification of butyrate-producing GPAC was unsatisfactory because it relied heavily on the enzyme profile; testing for indole production was of limited discriminative value. Most strains of $P$. asaccharolyticus and $P$. indolicus were very similar in enzyme profile, microscopic appearance and colonial morphology, but a sub-group of $P$. asaccharolyticus could be distinguished. A further indole-positive group corresponding to Hare group III was also noted. Strains of $P$. prevotii and $P$. tetradius were very similar, but easily distinguished from other butyrateproducing GPAC. However, $45 \%$ of the butyrate-producing cocci could not be assigned to recognised species; most of these were assigned to one of two new groups, the ADH group and the bGAL group, by their enzyme profile, microscopic appearance and smell. Four strains that produced a terminal VFA peak of isovaleric acid formed a new group designated 'ivoricus'. Reliable features for the identification of $P$. anaerobius were GLC (all GPAC that produced isocaproic acid were identified as $\boldsymbol{P}$. anaerobius), enzyme profile and sensitivity to SPS. Two clinical strains that produced caproic acid were identified as Hare group VIII; they were distinguished from Peptococcus niger by their enzyme profile and colonial morphology.

A phenotypic classification based on GLC and enzyme profile is presented, with a method for the identification of most strains of GPAC within $48 \mathrm{~h}$ of primary isolation.
\end{abstract}

\section{Introduction}

In posing the question "What are anaerobic cocci?", Watt and Jack" stated that "The absence of clear guidelines for characterisation (of gram-positive anaerobic cocci) ... has held back studies on the role of these organisms in health and disease".

Gram-positive anaerobic cocci (GPAC) are isolated from clinical specimens more often than any group of anaerobic bacteria except Bacteroides spp., ${ }^{2-4}$ yet many strains are still difficult or impossible to identify in the diagnostic laboratory. As a result, very little work has been done on the clinical importance of

Received 3 Aug. 1990; accepted 28 Sept. 1990

- Correspondence should be sent to I. J. Mitchelmore. individual species of GPAC. Present identification schemes rely mainly on gas-liquid chromatography (GLC) and carbohydrate fermentation reactions. ${ }^{5,6}$ However, many GPAC do not utilise carbohydrates, ${ }^{7,8}$ so species are distinguished mainly on the basis of negative results. Furthermore, the taxonomy is still disputed; the two most recent studies 9,10 disagree on many issues, for instance the relatedness of Peptostreptococcus anaerobius to other GPAC and the classification of the butyrate-producing cocci.

Ezaki and Yabuuchi ${ }^{11}$ showed that GPAC were easily differentiated by their amidase and oligopeptidase activities. This major step forward contributed to the development of commercial pre-formed enzyme kits for the identification of anaerobic cocci, testing for saccharolytic and proteolytic enzymes rather than for carbohydrate fermentation reactions. We exam- 
ined two kits for their ability to identify 69 clinical and 30 reference strains of GPAC, ${ }^{12,13}$ and found complete agreement between identification based on the pre-formed enzyme profile and grouping by GLC based on the scheme of Sutter et al. ${ }^{6}$

To clarify the key features for the identification of the recognised species of GPAC, and to detect groups of strains which might constitute undescribed species, we have studied a collection of clinical strains of GPAC and compared them with a reference collection that included all the recognised type strains of GPAC ${ }^{8}$ For the pre-formed enzyme kit, we selected ATB 32A, the commercially available form of the prototype API kit tested previously. ${ }^{12,13}$

\section{Materials and methods}

\section{Bacterial strains}

The clinical strains examined comprised 256 of a collection of 266 strains isolated in the Department of Medical Microbiology, St Bartholomew's Hospital,
London, in 1986-1987. They were stored in Robertson's Cooked Meat Broth (RCM; Southern Group Laboratories, Hither Green, London) at $-20^{\circ} \mathrm{C}$ until they were studied. They were then incubated anaerobically, in an atmosphere of $\mathrm{N}_{2} 80 \%, \mathrm{H}_{2} 10 \%, \mathrm{CO}_{2}$ $10 \%$, on enriched blood agar (Columbia Agar Base, Difco, with horse blood 6\%, supplemented with haemin $5 \mathrm{mg} / \mathrm{L}$ and vitamin $\mathrm{K} 11 \mathrm{mg} / \mathrm{L}$ ), for at least 10 days to check purity. Further checks on purity were done at every stage; colonial variants were subcultured and tested separately. Ten strains grew in an aerobic atmosphere with $\mathrm{CO}_{2} 6 \%$ after incubation for 1 week, or were resistant to metronidazole (as defined by growth up to a metronidazole $5-\mu \mathrm{g}$ disk after incubation for $48 \mathrm{~h}$ ). As they would not be termed 'anaerobic cocci' by the definition of Watt and Jack, ${ }^{1}$ they were not included in this study. The strains examined further were cocci or coccobacilli sensitive to vancomycin and metronidazole which grew only in an anaerobic atmosphere.

The clinical strains were compared with a collection of 39 reference strains (table I), which included the 10

Table I. Identity and synonyms of type and reference strains of GPAC

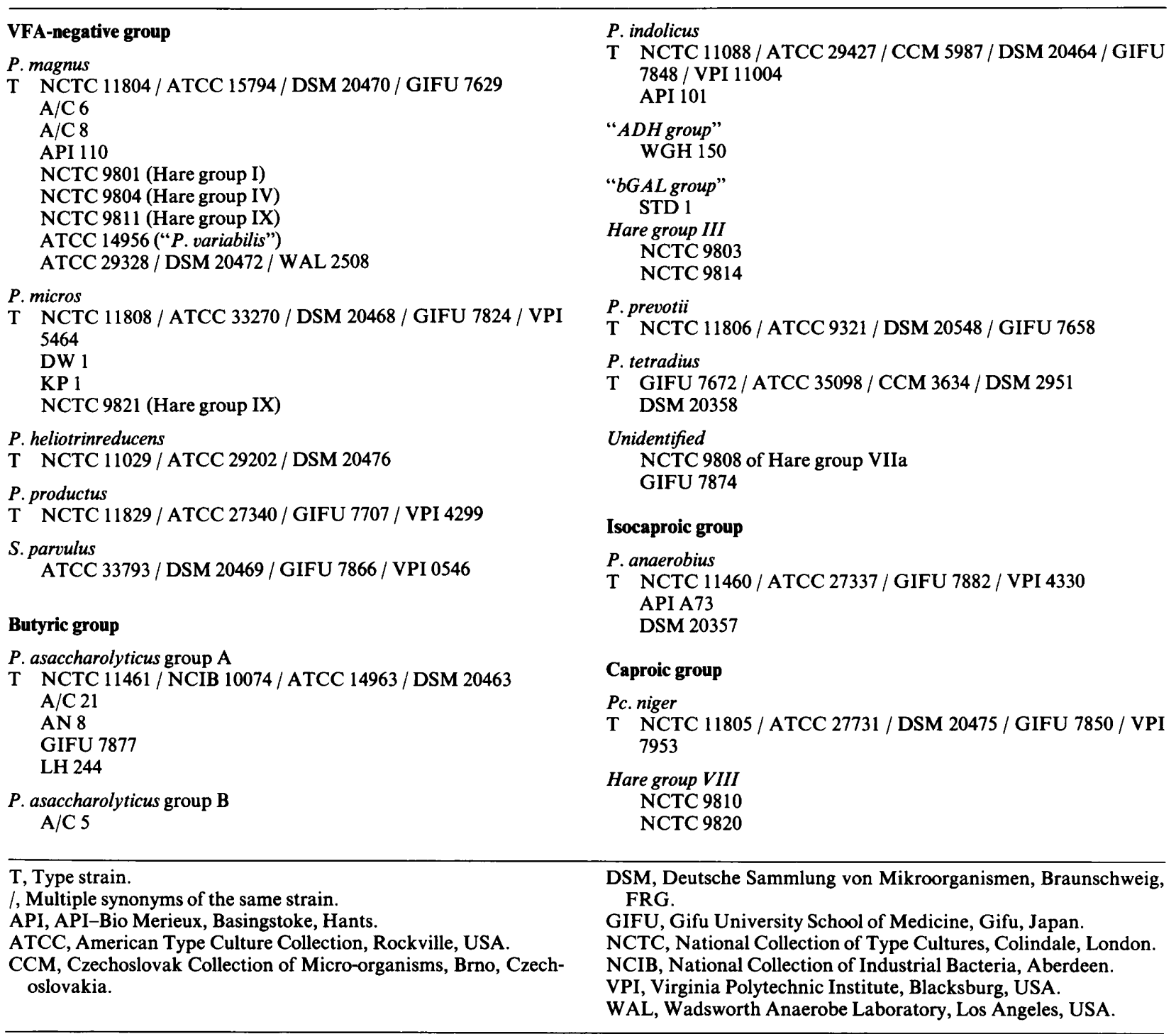


type strains of recognised species of $\mathrm{GPAC}^{8}$ and those previously studied by Murdoch et al. ${ }^{13}$ The type strain of Streptococcus parvulus was also included because it conforms to the definition of an anaerobic coccus. ${ }^{1}$

\section{Identification methods}

Colonial morphology and microscopy. The size, shape, colour and smell of colonies were assessed after incubation for $48 \mathrm{~h}$ and 5 days on enriched blood agar. Gram-stained smears were made after incubation for $48 \mathrm{~h}$ and examined for the size (and variation in size), shape, arrangement and degree of decolouration of the cells. Smears for microscopy were made from isolated colonies whenever possible, but this was not feasible for many of the slow-growing strains.

Production of volatile fatty acids and interpretation of results. Strains were incubated for $48 \mathrm{~h}$ in RCM broth and the supernate was examined for the presence of volatile fatty acids (VFAs) ${ }^{5}$ with a Perkin-Elmer Sigma 1 chromatograph. A $2 \cdot 5-\mu$ l volume of sample was injected into a column of internal measurements $6 \mathrm{ft} \times 0.25 \mathrm{inch}$, packed with SP 1220 (Supelco, Sawbridgeworth, Herts) on acid-washed chromosorb W (100-120 grade; Phase-Sep, Deeside, Chester). The oven temperature was $140^{\circ} \mathrm{C}$ and the carrier gas was oxygen-free nitrogen (BOC), at a flow rate of $25 \mathrm{ml} /$ min. VFAs were identified by comparison of retention times with a standard mixture of known VFAs, ranging from formic acid to heptanoic acid (Supelco). The run time was long enough to detect the production of heptanoic acid. Strains were grouped according to the terminal peak detected. As formation of acetic acid is not considered to be of value in identification, ${ }^{6}$ and small quantities were present in many of the batches of RCM broth used for GLC studies, strains which produced no VFAs or acetic acid alone were described as 'VFA-negative'.

Antibiotic sensitivity tests. Antibiotic sensitivity testing was performed by the disk method, with metronidazole 5- $\mu \mathrm{g}$, vancomycin 5- $\mu \mathrm{g}$ and novobiocin 5- $\mu \mathrm{g}$ disks (Mast Laboratories, Merseyside) and sodium polyanethol sulphonate (SPS) $1-\mathrm{mg}$ disks (Oxoid). Tests were done with a heavy growth inoculated on enriched blood agar. Zone diameters of $>15 \mathrm{~mm}$ for metronidazole and novobiocin and $>12 \mathrm{~mm}$ for vancomycin and SPS were accepted as representing sensitivity. The type strains of $P$. anaerobius and $P$. indolicus (NCTC nos. 11460 and 11088) were used for comparison to control the tests.

Determination of pre-formed enzyme profiles. Bacteria were inoculated on to fresh plates of enriched blood agar (more than one plate was used for slowly growing strains), and the plates were incubated anaerobically at $37^{\circ} \mathrm{C}$. Growth was harvested after $48 \mathrm{~h}$, to produce an inoculum of MacFarland standard 4 (estimated by eye). Pre-formed enzyme profiles were determined with a commercially available kit, ATB 32A (API-

Table II. Biochemical reactions of the ATB 32A pre-formed enzyme kit

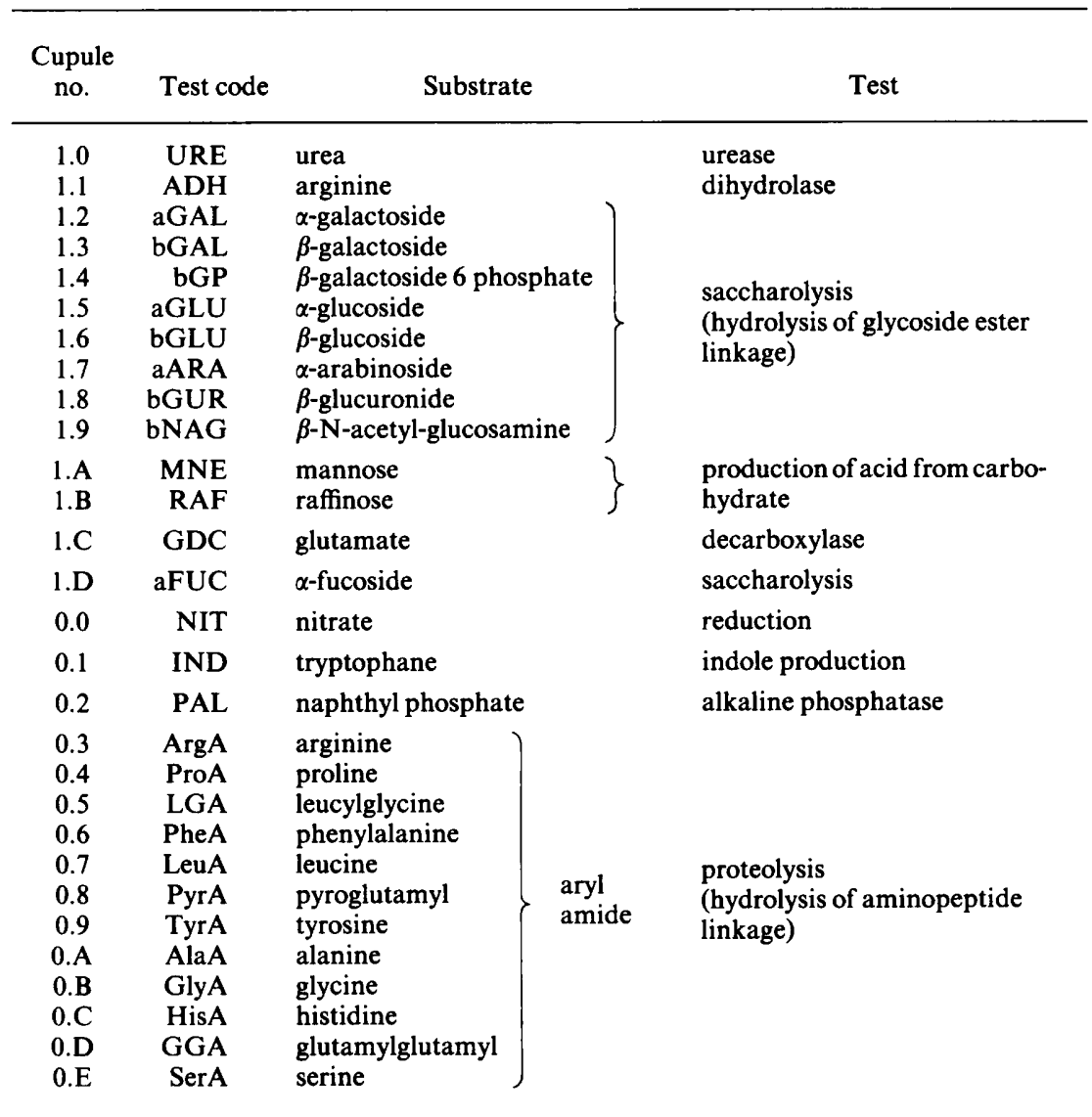


BioMerieux, Basingstoke, Hants), described as the "commercial kit"; the reactions tested are listed in table II. Using a colour chart supplied by the manufacturer, results were graded as negative, very weakly positive, weakly positive or strongly positive; if they were weakly or strongly positive, they were recorded as positive. Catalase production was determined by adding $\mathrm{H}_{2} \mathrm{O}_{2} \%$ to cupule 1 .D. Strains were identified by comparison with the enzyme profiles generated by type strains or reference strains. Kits employed during the development of the commercial kit were occasionally used to help to identify difficult strains; they are referred to in this report as "prototype kits". They have been described previously. ${ }^{12}$ They differed mainly in the inclusion of three tests for the production of acid from glucose, fructose and trehalose, and in the volume of inoculum (135 $\mu \mathrm{l}$ instead of $55 \mu \mathrm{l})$. The results obtained with the prototype kits were similar to those with the commercial kits, but individual proteolytic reactions were often stronger in the prototype kit, particularly for butyrate-producing cocci.

Coagulase production was examined with freshly reconstituted lyophilised rabbit plasma (Lab M, Bury, Lancs). Strains were inoculated into neat plasma and into plasma diluted 1 in 10 in distilled water, incubated in an anaerobic atmosphere at $37^{\circ} \mathrm{C}$, and checked for clot production after $4 \mathrm{~h}$ and $24 \mathrm{~h}$.

\section{Results}

The 256 clinical strains and 39 references strains of GPAC were allocated to five groups on the basis of VFA production (table III); $>95 \%$ of strains were placed in the VFA-negative, butyric or isocaproic groups. One strain gave inconsistent results and could not be placed in a VFA group. The strains were independently grouped by their pre-formed enzyme profiles. Apart from the single strain which could not be allocated to a VFA group, and three strains of $P$. anaerobius, there was very good consistency between the results of VFA grouping by GLC and the enzyme profile produced in the ATB 32A kit (table IV).

\section{VFA-negative group}

Sixteen reference strains and 111 clinical strains were VFA-negative. All except one of the clinical strains were identified by their enzyme profiles (table $\mathrm{V})$ as $P$. magnus, $P$. micros or $P$. heliotrinreducens; no clinical strains of $P$. productus or $S$. parvulus were identified.

$P$. magnus. Nine reference strains and 78 clinical strains were identified as $\boldsymbol{P}$. magnus by comparison of their enzyme profiles with that of the type strain. All strains produced similar proteolytic enzyme profiles: ArgA, LeuA and PyrA (arginine, leucine and pyroglutamyl aminopeptidases) results were always strongly positive, but ProA, PheA and GGA (proline, phenyl-
Table III. Grouping by VFA production of reference and clinical strains of GPAC

\begin{tabular}{l|rrrr}
\hline & \multicolumn{4}{|c}{ Number of strains } \\
\cline { 2 - 5 } VFA group & Type & $\begin{array}{c}\text { Other } \\
\text { reference }\end{array}$ & Clinical & Total \\
\hline VFA-negative & 5 & 11 & 111 & 127 \\
Butyric & 4 & 13 & 94 & 111 \\
Isovaleric & 0 & 0 & 7 & 7 \\
Isocaproic & 1 & 2 & 41 & 44 \\
Caproic & 1 & 2 & 2 & 5 \\
Ungrouped & 0 & 0 & 1 & 1 \\
$\quad$ Total & 11 & 28 & 256 & 295 \\
\hline
\end{tabular}

Table IV. Identification by pre-formed enzyme profile of reference and clinical strains of GPAC

\begin{tabular}{|c|c|c|c|c|}
\hline \multirow[b]{2}{*}{ Group } & \multicolumn{4}{|c|}{ Number of strains } \\
\hline & Type & $\begin{array}{l}\text { Other } \\
\text { reference }\end{array}$ & Clinical & Total \\
\hline \multicolumn{5}{|l|}{ VFA-negative group } \\
\hline P. magnus & 1 & 8 & 78 & 87 \\
\hline P. micros & 1 & 3 & 27 & 31 \\
\hline P. heliotrinreducens & 1 & 0 & 5 & 6 \\
\hline S.parvulus & 1 & 0 & 0 & 1 \\
\hline P.productus & 1 & 0 & 0 & 1 \\
\hline Unidentified & 0 & 0 & 1 & 1 \\
\hline \multicolumn{5}{|l|}{ Butyric group } \\
\hline P. asaccharolyticus & 1 & 5 & 44 & 50 \\
\hline P. indolicus & 1 & 1 & 2 & 4 \\
\hline “ADH” group & 0 & 1 & 18 & 19 \\
\hline “bGAL" group & 0 & 1 & 10 & 11 \\
\hline Hare groupIII & 0 & 2 & 7 & 9 \\
\hline P.prevotii & 1 & 0 & 0 & 1 \\
\hline P. tetradius & 1 & 1 & 5 & 7 \\
\hline Unidentified & 0 & 2 & 8 & 10 \\
\hline \multicolumn{5}{|l|}{ Isovaleric group } \\
\hline "ivoricus" group & 0 & 0 & 4 & 4 \\
\hline \multicolumn{5}{|l|}{ Isocaproic group } \\
\hline${ }^{*} P$. anaerobius & 1 & 2 & 44 & 47 \\
\hline \multicolumn{5}{|l|}{ Caproic group } \\
\hline Hare group VIII & 0 & 2 & 2 & 4 \\
\hline Ungrouped & 0 & 0 & 1 & 1 \\
\hline Total & 11 & 28 & 256 & 295 \\
\hline
\end{tabular}

* Three strains of $P$. anaerobius did not produce isocaproic acid and were, therefore, placed by their VFA profile in the isovaleric group.

alanine and glutamylglutamyl aminopeptidases) tests gave negative results. There was some variation with the other proteolytic enzymes, between strains and when the same strain was repeatedly tested, which may have been due to minor variations in the inoculum used. None of the strains showed any saccharolytic activity. Acid was not produced from mannose or raffinose, nor (when the prototype kit was used) from glucose or trehalose; however, seven $(22 \%)$ of the 32 strains tested with the prototype kit produced small amounts of acid from fructose. Alkaline phosphatase 
(PAL) production was usually negative or very weak, but six strains $(7 \%)$, including the type strain, gave positive results. Fourteen strains $(19 \%)$ produced catalase.

The microscopic appearance was always of cocci arranged in clumps, never in chains. A clump typically consisted of larger cocci in the centre and much smaller cocci on the periphery; they were decolourised little or not at all. Cells were always larger than those of $P$. micros, but the microscopic appearance was very variable and could not be used to separate strains of $P$. magnus from many strains of butyrate-producing cocci.

The colonial morphology of $P$. magnus was also highly variable. Most strains produced colonies of about $1 \mathrm{~mm}$ diameter after $48 \mathrm{~h}$, but three strains that grew much more slowly would not have been visible on primary culture at this stage. After incubation for 5 days, all strains produced colonies larger than $1 \mathrm{~mm}$, and $>90 \%$ produced colonies as large as $2 \mathrm{~mm}$. The colour was highly variable; colonies were most frequently translucent, but varied from white, glistening colonies highly suggestive of staphyloccoci, to greyish or yellowish colonies which could be confused with those of $P$. asaccharolyticus. The colour of many strains varied also from plate to plate. The surface could be glistening or matt. Colonies were always entire and circular, and usually low convex, similar to those of many other GPAC. One strain did not produce visible colonies for 3 days, and then formed domed, white colonies similar to those of $P$. micros (though without a halo, see below). Two strains were sometimes weakly $\beta$-haemolytic. None of the strains produced a detectable smell. All strains were resistant to novobiocin and SPS.

P. micros. Four reference and 27 clinical strains were identified as $P$. micros. All were easily distinguished from $P$. magnus by their very strong proteolytic activity: ProA, PheA and GGA tests were each positive with $>90 \%$ of strains. Most of the proteolytic reactions were always positive, the most frequent exceptions being LGA (leucylglycine aminopeptidase, eight strains) and AlaA (alanine aminopeptidase, six strains). All strains tested were very strongly PALpositive. None produced catalase or ADH (arginine dihydrolase), or showed any saccharolytic activity, but four produced GDC (glutamate decarboxylase).

Microscopic examination showed very small cocci which could always be distinguished from cells of $P$. magnus by their size. However, only five strains $(15 \%)$ were arranged mainly in chains; eight strains were in clumps and the others in both clumps and chains. Therefore, cell arrangement is not a reliable identification guide in secondary culture, though chain formation is more marked on primary isolation (I. Mitchelmore, personal observation).

Strains of $P$. micros grow very slowly on enriched blood agar; colonies were always $<1 \mathrm{~mm}$ in diameter after $48 \mathrm{~h}$, usually too small to be visible on primary culture. After 5 days, most strains produced very distinctive colonies: glistening white, domed, entire, circular, $1 \mathrm{~mm}$ in diameter, and surrounded by a yellow-brown halo of about $2 \mathrm{~mm}$. Two strains produced grey colonies also. Several strains produced variants of different colony size. Three strains did not form haloes and at least one other strain did not always produce a halo. None of the strains produced a detectable smell. Five strains sometimes showed a small zone of inhibition around the SPS disk (diameter $<12 \mathrm{~mm}$ ), but this was not reproducible, and the zone was always smaller than with strains of $P$. anaerobius. All strains were resistant to novobiocin.

$P$. heliotrinreducens. Five clinical strains were identified as $P$. heliotrinreducens by their microscopic appearance and enzyme profile. They were notable for their very slow rate of growth and sensitivity to novobiocin.

The enzyme profiles were highly consistent: tests for ADH and the proteolytic enzymes ProA, PheA and LeuA were always strongly positive, but those for PAL, LGA (leucylglycine aminopeptidase) PyrA, GGA and the saccharolytic enzymes were always negative. None of the strains produced catalase or acid from carbohydrates.

The microscopic appearance was of small cocci arranged in clumps and chains, often with many pleomorphic forms-notably a rod-shaped cell which looked like a contaminant. The clinical strains grew more slowly than strains of $P$. micros and the colonies were markedly different; only two strains produced visible individual colonies after $48 \mathrm{~h}$, but after 5 days, glistening, low convex, entire, circular, grey-pink colonies, $1 \mathrm{~mm}$ in diameter, could be seen. After 10 days, the colonies were $3-4 \mathrm{~mm}$ in diameter, flat, glistening and grey; they did not smell or form haloes. The type strain grew even more slowly; after 5 days, tiny, pink colonies were visible, which by 10 days were 1-2 mm in diameter, translucent, low convex, entire and circular. All strains were sensitive to novobiocin but resistant to SPS.

$S$. parvulus. The type strain of $S$. parvulus, ATCC 33793, was an obligately anaerobic gram-positive coccus, sensitive to metronidazole, which did not produce VFAs. The pre-formed enzyme profile was distinguished from that of $P$. micros by the production of acid from mannose (and, in the prototype kit, from glucose and fructose), and weak saccharolytic activity (notably $\beta$-galactosidase, bGAL). Catalase was not produced. Microscopic examination showed very small cocci arranged in clumps and chains. This strain was very slow-growing; colonies were hardly visible after $48 \mathrm{~h},<1 \mathrm{~mm}$ in diameter and grey-pink after 5 days, and 1-2 mm, grey, low convex, circular and entire with no smell after 10 days. It was sensitive to novobiocin but not to SPS.

$P$. productus. The type strain of $P$. productus, NCTC 11829 , was easily distinguished from all other GPAC studied; no clinical strains were identified as this species. It showed considerable saccharolytic activity (five of the eight enzyme tests gave positive results), 
Table V. Pre-formed enzyme profiles of GPAC obtained with the ATB 32A kit

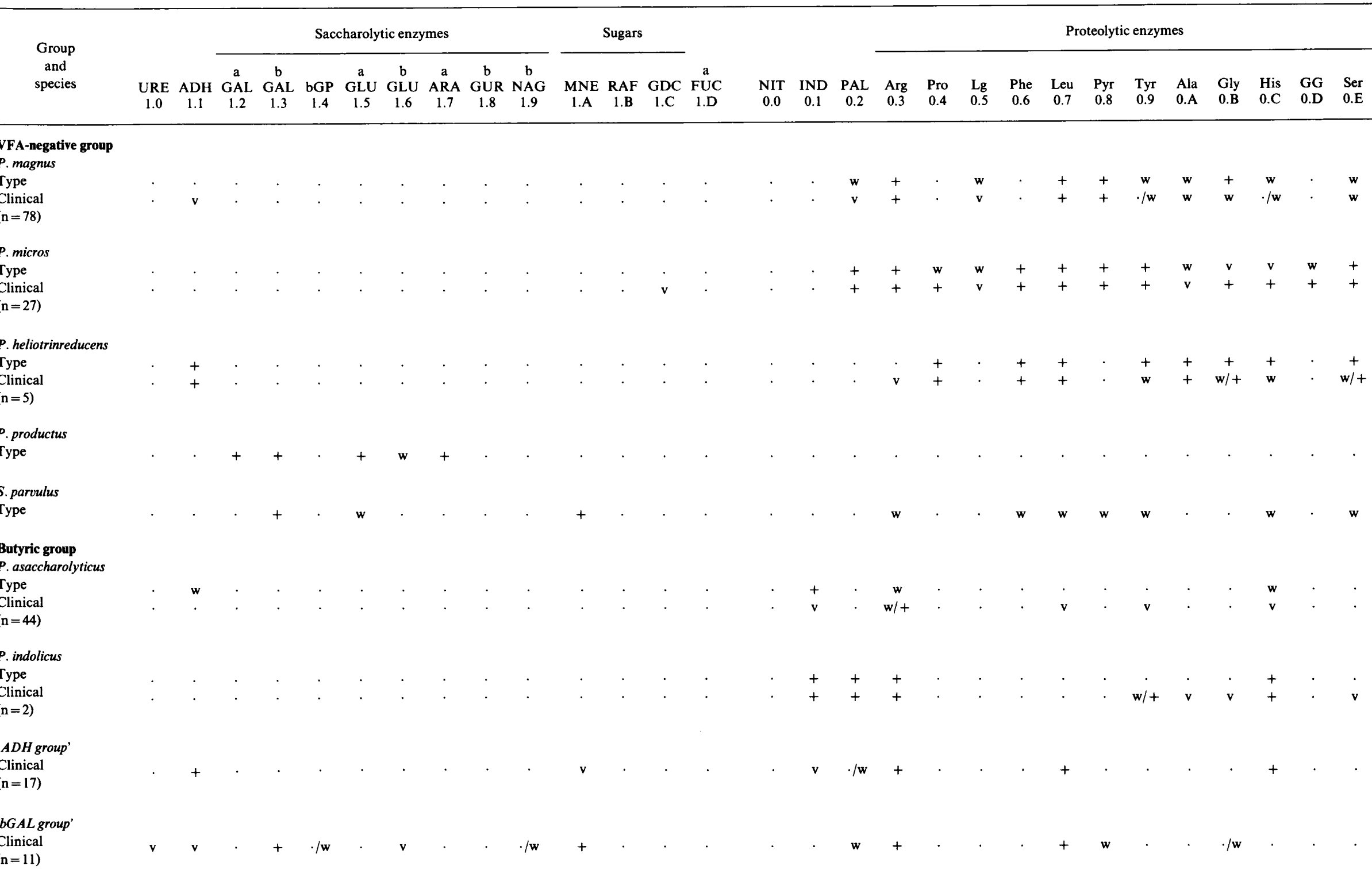




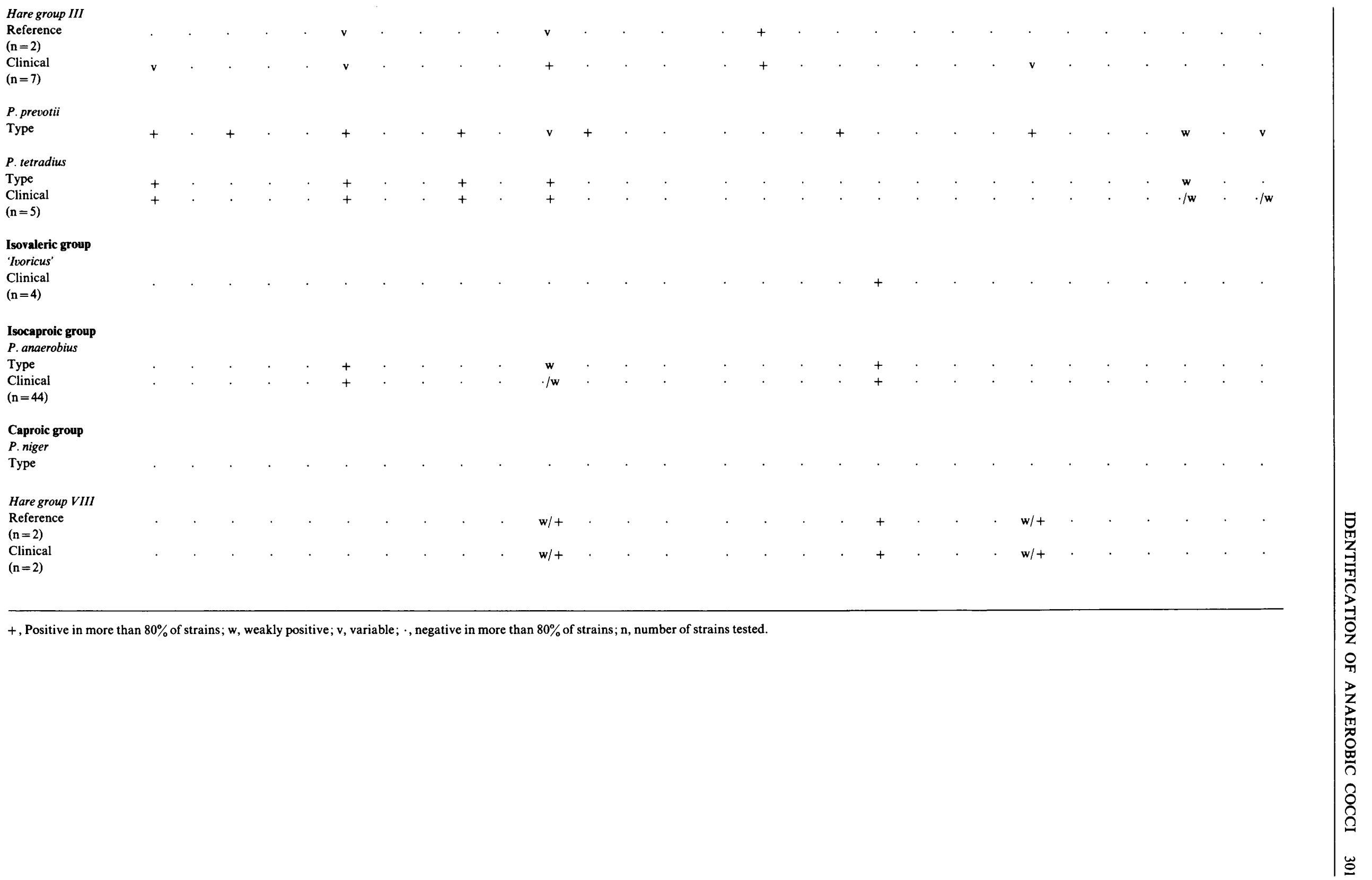


but acid was not produced from carbohydrates and no proteolytic activity was detected. Catalase was not produced. The microscopic appearance was of medium-sized, chunky cocci or coccobacilli arranged in clumps. The colonies were very unusual: after only $48 \mathrm{~h}$, they were $1-3 \mathrm{~mm}$ in diameter, grey-brown, glistening, circular and entire, with either a brown pigment diffusing into the agar or a form of haemolysis. This strain was sensitive to novobiocin but not to SPS, and did not smell.

$S B H 467$. One slow-growing, VFA-negative strain, SBH 467, could not be identified definitively. Microscopy showed very small cocci and the colonies were translucent and lacked haloes, but the pre-formed enzyme profile, though it showed strong proteolytic activity, was different from those of strains of $P$. heliotrinreducens and P. micros.

\section{Butyric group}

Butyric acid was the sole VFA formed by 94 clinical strains and 17 reference strains. This group was by far the most difficult to identify satisfactorily; the most effective method for differentiating strains was the enzyme profile, but the results of one important test, the production of acid from mannose, were not reproducible when the same strains were tested repeatedly.

$P$. asaccharolyticus. Six reference strains and 44 clinical strains were identified by their enzyme profiles as $P$. asaccharolyticus. They could be subdivided, most easily by their colonial morphology, into two groups, designated groups A and B. None of the strains showed any saccharolytic activity or produced acid from carbohydrates (in prototype or commercial kits). None produced urease or PAL; the type strain alone produced $\mathrm{ADH}$. Thirty-eight strains $(76 \%)$, including 33 clinical strains, gave strongly positive indole reactions and a further three gave weak or very weak reactions. Proteolytic activity in the commercial kit was limited to a few enzymes of which ArgA was the strongest. However, some strains showed little or no proteolytic activity. The prototype kit generated consistently stronger proteolytic reactions.

Thirty-nine strains (the type strain, four other reference strains and 34 clinical strains) were placed in group A. Thirty-four strains $(87 \%)$ in this group were strongly indole-positive. One strain produced catalase. After 48 h, 34 strains of group A produced colonies of $1 \mathrm{~mm}$ diameter or larger, and all strains were easily visible. By 5 days, all produced low convex, circular, entire colonies, $2 \mathrm{~mm}$ or larger, with a distinctive lemon-yellow tinge and a lighter central peak, a colony type seen otherwise only with strains of $P$. indolicus. Nine strains regularly, and four strains sometimes, produced a musty "mouse's cage" smell identical to that of Veillonella spp. No other GPAC studied produced this smell. The microscopic appearance was also distinctive-medium or medium-small cocci, with little size variation, arranged in clumps but not overlapping, like a mosaic. The smears tended to decolourise very markedly (as with $P$. indolicus), and occasionally the cocci appeared gram-negative and closely resembled Neisseria spp.; however, all strains were sensitive to vancomycin. The type strain was sensitive to novobiocin, otherwise all strains were resistant to SPS and novobiocin.

The 11 strains (one reference strain and 10 clinical strains) placed in group B grew more slowly; none produced colonies as large as $1 \mathrm{~mm}$ by $48 \mathrm{~h}$, and four were hardly visible. By 5 days, colonies were $1 \mathrm{~mm}$ in diameter, entire, flat, translucent, usually with a matt surface, resembling some strains of $P$. magnus. Two strains produced an unpleasant smell similar to that of hare group III or the ADH group, but none produced a musty smell. Microscopy showed considerable variation in the size and shape of the cocci, many of which were oval or sometimes elliptical. The proteolytic enzyme reactions were generally weaker than those of group A. Six strains $(55 \%)$ produced catalase. Only four strains $(36 \%)$ were strongly indole-positive; three further strains were weakly positive and four were indole-negative. All were resistant to SPS and novobiocin.

$P$. indolicus. Two clinical strains closely resembled the two reference strains of $P$. indolicus, and were tentatively distinguished from $P$. asaccharolyticus by their ability to produce PAL, their unpleasant smell and slightly different colonial morphology.

The enzyme profiles were similar to those of strains of $P$. asaccharolyticus, but the positive reactions were stronger: results for $\operatorname{ArgA}$, TyrA (tyrosine aminopeptidase) and HisA (histidine aminopeptidase) were positive, SerA and GlyA (serine and glycine aminopeptidase) variable. All strains produced indole. The test for PAL always gave a strongly positive result. None of the strains produced catalase or reduced nitrate.

The microscopic appearance was of medium to small cocci, usually with some size variation, arranged in loose clumps. They were decolourised markedlysometimes no gram-positive cocci could be foundbut all were sensitive to vancomycin. Three of the strains were fast-growing, forming 1-2-mm, greywhite colonies after only $48 \mathrm{~h}$; one clinical strain grew more slowly but its colonies were still visible. After 5 days, all strains formed distinctive, raised, yellow-buff colonies, 3-4 $\mathrm{mm}$ in diameter, with an unpleasant smell similar to that of Hare group III or many strains of the ADH group. The colonies varied markedly in size. Both reference strains and one clinical strain were sensitive to novobiocin; all were resistant to SPS. Both reference strains, but neither clinical strain, produced coagulase, but only in neat serum after overnight incubation. Strains from all of the other groups of butyrate-producing cocci were tested and were consistently coagulase-negative.

The $A D H$ group. One reference strain and 18 clinical strains were grouped together by their enzyme profile, notably by the production of $\mathrm{ADH}$. The production of 
acid from carbohydrates was variable. Many strains produced a distinctive, unpleasant smell.

Results of tests for ADH and three proteolytic enzymes, ArgA, LeuA and HisA, were strongly positive, but those of all other proteolytic and saccharolytic tests were negative except for one strain which produced bGUR ( $\beta$-glucuronidase). Two strains $(11 \%)$ were strongly indole-positive. Five strains $(25 \%)$ produced catalase. Acid was usually produced from mannose, but this test did not always give reproducible results when the same strains were re-tested, and three strains gave consistently negative results. Seven strains were examined with the prototype kit to test its wider range of carbohydrate substrates; five produced acid from glucose, mannose and fructose, and two showed little or no activity. Thus, strains of this group produced similar profiles to those of strains of $P$. asaccharolyticus and $P$. indolicus, but could be most easily separated by the production of ADH. The microscopic appearance was usually of medium-large to small cocci in clumps, with little decolouration; there were sometimes many tetrads. The colonies were up to $1 \mathrm{~mm}$ or slightly smaller by $48 \mathrm{~h}$ (except for the reference strain which grew more slowly). After 5 days, they were usually $2-3 \mathrm{~mm}$, grey-white, low convex, entire and circular. Seven strains consistently, and six more variably, produced an unpleasant smell. All strains were resistant to novobiocin and SPS.

The $b G A L$ group. One group of indole-negative, butyrate-producing cocci was tentatively separated by the consistent ability to produce acid from carbohydrates and the weak but consistent production of certain saccharolytic enzymes, notably bGAL, and PAL.

One reference strain and 10 clinical strains were placed in this group. All produced acid from mannose but not from raffinose; of five tested with the prototype kit, all produced acid from glucose, mannose and fructose, and three from trehalose also. All strains except one produced bGAL, though sometimes weakly, and usually bGP ( $\beta$-galactosidase-6-phosphate) bGLU ( $\beta$-glucosidase) and bGUR weakly. The exception was the reference strain, which produced bGLU strongly but not other saccharolytic enzymes. Results of tests for PAL and PyrA were consistently weakly positive, those for ArgA and LeuA were strongly positive and the other proteolytic reactions were weak or negative. None produced catalase. The microscopic appearance was usually of medium-large to small clumps of cocci. One strain consistently produced medium-sized cocci only. Colonies were about $1 \mathrm{~mm}$ diameter after $48 \mathrm{~h}$; after 5 days, they were 1-2 mm, grey, flat or low convex, entire, circular, often matt, with whiter centres. They varied markedly in size and shape. They did not smell. Three strains gave small zones of inhibition around the SPS disk (< $12 \mathrm{~mm}$ ), but all were resistant to novobiocin.

Hare group III. Seven clinical strains gave profiles very similar to strains NCTC 9803 and 9814 of Hare group III. Strains were identified by the production of indole but not of proteolytic enzymes, except for three weakly indole-positive strains which were strongly PyrA positive. Three strains consistently produced urease; a fourth strain was tested three times and gave a positive result once. All produced acid from mannose, and, with the prototype kit, from glucose and fructose. Two strains produced aGLU ( $\alpha$-glucosidase). Other saccharolytic enzymes and catalase were not produced. Microscopy showed strongly staining cocci arranged in clumps, with marked size variation similar to strains of $P$. magnus and the ADH group. Diplococci and tetrads, arranged with the opposing surfaces flattened or concave, similar to meningococci, were frequently seen. After $48 \mathrm{~h}$, the PyrA-negative strains produced colonies about $1 \mathrm{~mm}$ diameter, whereas the PyrA-positive strains produced smaller but still visible colonies. After 5 days, colonies were usually $2-3 \mathrm{~mm}$, grey with whiter centres, mucoid, entire and circular; they sometimes resembled colonies of staphylococci. All but one strain produced an unpleasant smell similar to that produced by the ADH group and $\boldsymbol{P}$. indolicus. All strains were resistant to SPS and novobiocin.

The prevotii/tetradius group. Eight strains (the type strains of $P$. prevotii and $P$. tetradius, reference strain DSM 20358, and five clinical strains) produced very similar enzyme profiles, easily separated from all other GPAC by their saccharolytic activity. They also consistently produced urease. The type strains were not easy to distinguish, but strain DSM 20358 and at least four clinical strains were identified as $P$. tetradius.

All eight strains produced urease, aGLU and bGUR, a unique combination. The type strain of $P$. prevotii also produced aGAL ( $\alpha$-galactosidase), and one clinical strain was weakly bGLU-positive. None produced indole. The carbohydrate utilisation reactions were not always reproducible when the same strain was re-tested, but acid was usually produced from glucose, fructose and mannose. The type strain of $P$. prevotii differed in producing acid from raffinose but only weakly from the other sugars. Results of the proteolytic tests were usually negative or very weak when the commercial kit was used, but the prototype kit gave reproducible reactions with most strains: tests for ArgA, HisA, TyrA and SerA were strongly positive, and those for PheA, GlyA, AlaA and LeuA were weakly positive. One clinical strain was strongly PyrApositive. The type strain of $P$. prevotii gave strongly positive ArgA, PyrA and HisA reactions in the commercial kit. The type strain of $P$. prevotii and one clinical strain produced catalase. The microscopic appearance was of strongly staining cocci arranged in clumps; tetrad formation was prominent in only one strain, a clinical isolate. The cocci always varied markedly in size, with large to medium-large cocci in the centre of clumps and much smaller cocci on the periphery. Colonies were small but visible (diameter $<1 \mathrm{~mm}$ ) after $48 \mathrm{~h}$; by 5 days, they were usually $2 \mathrm{~mm}$ in diameter, matt grey with whiter centres, low convex, circular and entire. Some strains showed marked 
variation in size and shape. None produced any smell. All were resistant to SPS and novobiocin.

A feature unique to this group was the appearance of what were presumed to be sub-colonies after prolonged incubation (for at least a week): isolated colonies 3-4 mm across developed several lighter shiny protrusions $(<0.5 \mathrm{~mm})$ on their surfaces. This was noted consistently with three clinical strains and irregularly with the type strain of $P$. tetradius.

Other butyrate-producing GPAC. Two reference strains and eight clinical strains could not be placed in the above groups. Two strains were very similar: their enzyme profiles were almost identical, and microscopy showed small cocci, similar in size to $P$. micros. Five other clinical strains produced unique enzyme profiles. The final strain was isolated with and formed satellites round a strain of $P$. magnus; it was only suspected because this strain of $P$. magnus appeared to produce butyric acid. It grew very slowly in the presence of $P$. magnus (diameter $c .1 \mathrm{~mm}$ at 5 days) and has not yet been isolated in pure culture.

\section{Isovaleric group}

Seven clinical strains produced butyric acid and larger quantities of isovaleric acid, but not isocaproic acid. None of the reference strains produced this unusual VFA profile. Three strains were identified as $P$. anaerobius by their microscopic appearance, enzyme profile and sensitivity to SPS (see below). The remaining four strains formed a homogeneous group, described here as 'ivoricus' (iv being the shorthand VFA notation for isovaleric acid).

The 'ivoricus' group. All four strains produced a distinctive pre-formed enzyme profile: the test for ProA gave a strongly positive reaction and all other tests were negative. Microscopy showed clumps of cocci, which were very large in the middle of the clump and medium-sized round the edge, and which did not decolourise. Colonies were still tiny after incubation for $48 \mathrm{~h}$, but by 5 days they were $1-2 \mathrm{~mm}$, yellow-white, low convex, entire and circular. They did not smell. They were resistant to novobiocin, but two strains were sometimes (but not reproducibly) sensitive to SPS.

\section{Isocaproic group}

The type strain of $P$. anaerobius, two other reference strains and 41 clinical strains produced large quantities of isocaproic acid, and smaller, variable quantities of shorter-chain VFAs (propionic, isobutyric, butyric, isovaleric and valeric acids). All the GPAC tested which produced a major, terminal peak of isocaproic acid were identified as $P$. anaerobius.

$P$. anaerobius. Of the 47 strains of $P$. anaerobius, 44 $(95 \%)$ produced isocaproic acid; three clinical strains produced a major peak of isovaleric acid but no isocaproic acid. In the pre-formed enzyme profile, all produced aGLU and $92 \%$ were strongly ProA-positive.
Weak acid production from mannose was sometimes seen with the commercial kit; all of the 18 strains tested with the prototype kit also produced acid from glucose and fructose. Negative results were obtained in all other tests. Catalase was not produced. The microscopic appearance was usually highly pleomorphic, with a fascinating variety of shapes present, but with medium to medium-small coccobacilli arranged in chains predominating -an appearance unlike any other strains of GPAC studied. Some strains were more pleomorphic than others. Three strains comprised cocci rather than coccobacilli; in one strain, the cocci were consistently arranged in clumps. Colonies were grey and $1 \mathrm{~mm}$ diameter after $48 \mathrm{~h}$, and 2-3 mm, grey, flat, entire, circular or oval, with slightly raised whitish centres, after 5 days. Two strains produced atypical, raised white colonies. Fortytwo strains produced a distinctive plasticine smell, usually after $48 \mathrm{~h}$, which was diagnostic of $P$. anaerobius. Five strains consistently did not produce this smell, including the three strains that did not produce isocaproic acid. All strains were sensitive to SPS. Forty-four strains were sensitive to novobiocin.

\section{Caproic group}

None of the clinical strains was identified as Peptococcus niger, the only recognised species of GPAC that produces caproic acid. However, two reference strains of Hare group VIII and two very similar clinical strains all produced caproic acid, but in variable quantities.

$P c$. niger. The type strain produced large quantities of butyric, isovaleric and caproic acids, but showed no activity in the pre-formed enzyme kits. It did produce catalase. Microscopy showed medium to small cocci in clumps which did not decolourise. The colonies grew very slowly; they were almost invisible after $48 \mathrm{~h}$, but after 5 days formed $1 \mathrm{~mm}$, grey, raised, circular, entire colonies. They were not pigmented and did not smell. This strain was sensitive to novobiocin but not to SPS.

Hare group VIII. Two reference strains and two clinical strains produced large amounts of butyric acid and smaller quantities of isovaleric and caproic acids. The commercial kit showed production of acid from mannose and strongly positive ProA and weak PyrA reactions; results of other tests were always negative. When the prototype kit was used, acid was produced from glucose, fructose and mannose, but not from raffinose or trehalose. One strain produced catalase.

Microscopy showed medium to medium-small cocci that formed clumps and did not decolourise. The colonies were visible but small after $48 \mathrm{~h}$, and 1-2 mm, yellow-white, glistening, circular, entire and usually raised after 5 days. They did not smell. All strains were resistant to SPS and novobiocin.

$S B H$ 122. Production of VFAs by this strain was not consistent: no VFAs, or butyric acid alone, or butyric acid and small amounts of caproic acid, were 
produced on different occasions. The enzyme profile and microscopic appearance were consistent with identification as Hare group VIII, but the colonial morphology was not. This strain could not be satisfactorily identified.

\section{Discussion}

This study has shown almost complete agreement ( $>99 \%$ ) between grouping by VFA and by pre-formed enzyme profiles, and good correlation with the microscopic appearance and colonial morphology. We have been able to place $246(96 \%)$ out of 256 clinical strains of GPAC in 12 groups. However, a taxonomic study to test our results is essential, as the ADH, bGAL and "ivoricus" groups, accounting for 32 clinical strains, were created in this study, and Hare group III and Hare group VIII are not recognised taxa. We did not identify any clinical strains of two, perhaps three, of the 10 recognised species of GPAC (P. productus, Pc. niger and possibly $P$. prevotii), nor of $S$. parvulus.

\section{Assessment of the techniques used in this study}

GLC is accepted as a reliable technique for grouping anaerobic bacteria, ${ }^{5,6}$ and it was very useful in this study as a "gold standard" against which to test the reliability of pre-formed enzyme profiles. However, it is not sufficiently discriminatory: $>95 \%$ of strains belonged to one of three VFA groups (VFA-negative, butyric and isocaproic). The equipment required is expensive and not always available in routine laboratories. Thus, GLC is of limited use. Non-volatile fatty acids were not studied because their production by GPAC appears to be very limited ; 6 also, RCM usually contains lactic acid.

The pre-formed enzyme profile was the most effective technique used in this study because it discriminated well between strains; however, it was essential to use the recommended inoculum density. There was no overlap between the enzyme profiles of GPAC from different VFA groups, except for the three strains of $P$. anaerobius that did not produce isocaproic acid. Most individual tests were highly reproducible when the same strain was tested repeatedly. The results of the proteolytic enzyme reactions for the $P$. prevotii/tetradius group were variable, but these were not critical for the identification of this group. More importantly, the production of acid from mannose was not reproducible. This test may be important in separating $P$. asaccharolyticus and $P$. indolicus, species that do not utilise carbohydrates, ${ }^{7,8}$ from the newly created ADH and bGAL groups. The prototype kit was better than the commercial kit in this respect because it included tests with additional carbohydrates (glucose, fructose and trehalose).

Much practice was needed to achieve reproducible assessments from the same gram-stained smear, and repeated smears from the same strain did not always give the same results - the variation in size of the cocci and degree of decolouration were particularly liable to vary. However, the microscopic appearance of several groups was distinctive: the medium-sized cocci $(P$. asaccharolyticus group A and $P$. indolicus); the smallcelled VFA-negative group ( $P$. micros, $P$. heliotrinreducens and $S$. parvulus); and $P$. anaerobius. The microscopic appearance also supported the identification by enzyme profile of strains of Hare group VIII and $P$. magnus.

The colonial morphology was sometimes a very useful guide. Most strains of $P$. micros produced unique colonies. Several other groups, notably $P$. asaccharolyticus group A and $P$. anaerobius, could be presumptively identified when the colonial morphology and smell were taken together. A distinctive 'plasticine' smell was produced by $90 \%$ of $P$. anaerobius strains. Many strains of $\boldsymbol{P}$. asaccharolyticus group A produced a musty smell. An unpleasant smell was produced by $P$. indolicus, most strains of Hare group III and the ADH group, and some strains of $P$. asaccharolyticus group B.

Antibiotic sensitivity testing was of limited use. Sensitivity to metronidazole excluded anaerobic staphylococci or capnophilic streptococci, but it had to be checked at $48 \mathrm{~h}$ as many GPAC produced colonies which grew up to the metronidazole disk after prolonged incubation. This phenomenon, which was noted frequently with certain species, particularly $P$. magnus and $P$. asaccharolyticus, but never with strains of $P$. anaerobius or $P$. micros, should be studied further. Vancomycin sensitivity testing was particularly useful as evidence that a strain was gram-positive for strains of $P$. asaccharolyticus or $P$. indolicus which were decolourised completely in Gram's staining method; however, this test is not infallible because some strains of Bacteroides are sensitive to vancomycin. All strains of $P$. anaerobius in this study were sensitive to SPS, but so were two strains of the 'ivoricus' group; some strains of $P$. micros and the bGAL group showed small zones of inhibition with a few colonies growing up to the disk. Sensitivity to novobiocin was shown by only $95 \%$ of strains of $P$. anaerobius, and by strains of several other groups; therefore, it is of limited use for the presumptive identification of strains of $P$. anaerobius, as has been proposed by Wren et al. ${ }^{14}$

\section{Identification of groups of GPAC}

Standard manuals ${ }^{5,6}$ for the identification of anaerobic bacteria have characterised strains of the VFAnegative GPAC $P$. magnus and $P$. micros on the basis of negative results, relying on cell size alone to distinguish them; they have not included $P$. heliotrinreducens and $S$. parvulus. Other identification methods proposed have included protein electrophoresis, ${ }^{15} \mathrm{a}$ method which is not realistic in a routine diagnostic laboratory, and the production of $\mathrm{PAL},{ }^{8}$ for which no supporting data were provided in the accompanying text. In this study, the VFA-negative GPAC were 
most reliably identified by their pre-formed enzyme profiles, the key reactions being $\mathrm{ADH}, \mathrm{PAL}$, and ProA, PheA and PyrA. PAL production on its own was of limited value: it was formed by all strains of $P$. micros tested, but also by $7 \%$ of $P$. magnus strains. Cell size was a reliable distinguishing feature: all 87 largecelled, VFA-negative GPAC in this study (and all those studied by Ezaki et al. ${ }^{9}$ ) were identified as $P$. magnus. The other methods examined for the identification of strains of $P$. magnus were of little use. Colonial morphology was so variable that it was more likely to lead to misidentification; it was further complicated by the frequency of colonial variants, which could differ in shape, colour or size. Antibiotic sensitivity testing was of no assistance.

Strains of $P$. heliotrinreducens and $S$. parvulus were best separated from $P$. micros by enzyme profile, colonial morphology and sensitivity to novobiocin (table VI). Strains of $S$. parvulus also form lactic acid when Tween 80 is added to the medium, ${ }^{6}$ but nonvolatile fatty acid production was not tested in this study. The type strain of $P$. productus was very distinctive: the colonies were fast-growing and the strongly saccharolytic enzyme profile was unique. Its microscopic appearance was so unusual that it might lead to its presumptive identification as a grampositive rod.

Standard identification manuals ${ }^{5,6}$ separate butyrate-producing GPAC into indole-positive ( $P$. asaccharolyticus and $P$. indolicus) and indole-negative groups ( $P$. prevotii and $P$. tetradius), a distinction which other studies $9,11,13,16$ have shown to be questionable. Nucleic acid homology studies ${ }^{9,10}$ and previous studies of the pre-formed enzyme profiles ${ }^{11-}$ ${ }^{13}$ have shown that this group is very heterogeneous. In this study, indole-producing strains included all strains of Hare group III, two of the ADH group and

Table VI. Comparison between $P$. micros and P. heliotrinreducens

\begin{tabular}{|c|c|c|}
\hline Number of strains & $\begin{array}{l}\text { P. micros } \\
(31)\end{array}$ & $\begin{array}{c}\text { P. heliotrinreducens } \\
\text { (6) }\end{array}$ \\
\hline \multicolumn{3}{|l|}{ Similarities } \\
\hline Gram's strain & Tiny cocci & Tiny cocci/coccobacilli \\
\hline Growth & Slow & Very slow \\
\hline $\begin{array}{l}\text { VFAs detected by } \\
\text { GLC }\end{array}$ & Acetate/none & Acetate/none \\
\hline $\begin{array}{l}\text { Saccharolytic activ- } \\
\text { ity }\end{array}$ & None & None \\
\hline Proteolytic activity & Very strong & Strong \\
\hline \multicolumn{3}{|l|}{ Differences } \\
\hline Colonies & White, raised & Grey-pink, flatter \\
\hline Yellow-brown halo & Present in $90 \%$ & Absent \\
\hline $\begin{array}{l}\text { Alkaline phospha- } \\
\text { tase activity } \\
\text { (PAL) }\end{array}$ & Very strong & None \\
\hline $\begin{array}{l}\text { Arginine dihydro- } \\
\text { lase activity } \\
\text { (ADH) }\end{array}$ & None & Very strong \\
\hline $\begin{array}{l}\text { Novobiocin }(5-\mu \mathrm{g} \\
\text { disk) }\end{array}$ & Resistant & $\begin{array}{l}\text { Sensitive (zone diameter } \\
\quad 20-30 \mathrm{~mm})\end{array}$ \\
\hline
\end{tabular}

two unidentified strains, whereas $20-30 \%$ of strains of $P$. asaccharolyticus were indole-negative. We conclude that testing for indole production is of limited value.

We propose a phenotypic classification (table VII) which is still not satisfactory for butyrate-producing strains: it relies too heavily on one technique, the enzyme profile, in which strains of several groups gave very few positive reactions, and one important reaction, the production of acid from mannose, was not reproducible when the same strain was tested repeatedly. The identification criteria proposed must be checked by other methods, such as protein electrophoresis, and a study of the biochemistry of these groups is needed to determine more discriminatory tests.

Of the presently recognised species in the butyrate group, 34 clinical strains could be identified confidently as $\boldsymbol{P}$. asaccharolyticus group A because they had the same enzyme profile, microscopic appearance and colonial morphology as the type strain. Eleven strains were separated into $P$. asaccharolyticus group B because their colonial morphology, and to a lesser extent microscopic appearance, were different; they were also more slow-growing. This is in agreement with the results of Ezaki et al., ${ }^{9}$ who found three distinct nucleic acid homology groups in 18 strains identified as $P$. asaccharolyticus by the scheme of

Table VII. Comparison of the present classification of GPAC and a phenotypic classification based on this study

\begin{tabular}{|c|c|}
\hline $\begin{array}{l}\text { Present classification } \\
\text { (Holdeman Moore } \\
\text { et al. })^{8}\end{array}$ & $\begin{array}{c}\text { Phenotypic classification } \\
\text { based on this study }\end{array}$ \\
\hline $\begin{array}{l}\text { In genus } \\
\text { Peptococcus: } \\
\text { Pc. niger }\end{array}$ & $\begin{array}{l}\text { In genus } \\
\text { Peptococcus (G + C } 51 \mathrm{~mol} \%) \text { : } \\
\quad \text { Pc. niger }\end{array}$ \\
\hline $\begin{array}{l}\text { In genus } \\
\text { Peptostreptococcus: } \\
P . \text { anaerobius } \\
P . \text { asaccharolyticus } \\
P . \text { heliotrinreducens } \\
P . \text { indolicus } \\
P . \text { magnus } \\
P . \text { micros } \\
P . \text { prevotii } \\
P . \text { productus } \\
\text { P. tetradius }\end{array}$ & $\begin{array}{l}\text { In genus } \\
\text { Peptostreptococcus }(\mathrm{G}+\mathrm{C} \text { of recognised } \\
\text { species } 28-34 \text { mol\%): } \\
\text { VFA-negative group: } \\
\text { P. magnus } \\
\text { P. micros } \\
\text { P. heliotrinreducens } \\
\text { Butyrate-producing group: } \\
\text { P. asaccharolyticus } \\
\text { P. indolicus } \\
\text { P. prevotii } \\
\text { P. tetradius } \\
\text { Hare group III } \\
\text { "ADH" group } \\
\text { "“bGAL"group } \\
\text { + undescribed groups } \\
\text { Isovalerate-producing group: } \\
\text { "ivoricus" group } \\
\text { Isocaproate-producing group: } \\
\text { P. anaerobius } \\
\text { Caproate-producing group: } \\
\text { Hare group VIII }\end{array}$ \\
\hline $\begin{array}{l}\text { In genus } \\
\text { Streptococcus: } \\
\quad \text { S.parvulus }\end{array}$ & $\begin{array}{l}\text { Species incertae sedis }(\mathrm{G}+\mathrm{C} 45-46 \mathrm{~mol} \%) \\
\text { 'S.parvulus' } \\
\text { 'P.productus' }\end{array}$ \\
\hline
\end{tabular}


Table VIII. Reliability of identification features for strains of $P$. anaerobius

\begin{tabular}{ll}
\hline \multicolumn{1}{c}{ Feature } & Number of organisms $(\mathbf{n}=47)$ \\
\hline Sensitive to SPS & 47 \\
$\alpha$-GLU produced & 47 \\
Isocaproic acid produced & 44 \\
Sensitive to novobiocin & 44 \\
ProA produced & 43 \\
Colonies smell of plasticine & 42 \\
\hline
\end{tabular}

Holdeman et al. ${ }^{5}$ Two strains that produced PAL and an unpleasant smell were identified tentatively as $P$. indolicus, but not by the identification criteria used by Ezaki et al:: both reference strains examined, but neither of the clinical strains, produced coagulase, and none of the four strains reduced nitrate in the ATB 32A kit.

Five clinical strains were placed by their distinctive enzyme profile in the $P$. tetradius/prevotii group. Ezaki et $a l .{ }^{9}$ found close relationships between the type strains of $P$. prevotii and $P$. tetradius, but considerable differences between these strains and other indolenegative, butyrate-producing cocci. They differentiated $P$. prevotii from $P$. tetradius particularly by production of aGAL and weaker acid production by $P$. prevotii-identification features which we confirmed. At least four of the clinical strains in this study were identified as $P$. tetradius; like Ezaki et al., ${ }^{9}$ we have not found any clinical strains of GPAC which we could identify with certainty as $P$. prevotii.

Of the 94 clinical strains in the butyrate-producing group, $43(45 \%)$ did not belong to any of the recognised species; 28 were placed into one of two new groups, the ADH and bGAL groups, primarily on the basis of their enzyme profiles. Strains of Hare group III produced indole and acid from several carbohydrates, but showed no proteolytic or saccharolytic activity. They could be confused with some strains of $P$. asaccharolyticus which showed very little proteolytic activity. Two of the remaining strains were very similar to each other and would probably form another new group; the others were also separated from all other GPAC studied by their enzyme profile. Some of these strains might belong to other recognised groups of GPAC such as Coprococcus spp. ${ }^{8,17}$ the type strains of which were not available for study.

Most strains of $\boldsymbol{P}$. anaerobius were easily identified by various features (table VIII). Three strains did not produce isocaproic acid or a plasticine smell; Holdeman et al., ${ }^{5}$ but not Sutter et al., ${ }^{6}$ recognise this variation in the VFA profile. Microscopy of several strains showed medium-sized cocci, sometimes in clumps, rather than in chains. Members of the 'ivoricus' group grew slowly and did not produce aGLU; apart from the three strains of $P$. anaerobius mentioned above, they produced unique VFA and enzyme profiles.

The clinical strains of caproate-producing cocci were identified as Hare group VIII by their enzyme profile, supported by microscopic appearance and colonial morphology. This group could be confused with the 'ivoricus' group, but can probably be most easily separated by the production of caproic acid, acid production from mannose and weak production of PyrA. The type strain of Pc. niger was very slowgrowing, sensitive to novobiocin and produced catalase but no pre-formed enzymes. No clinical strains were identified.

\section{Proposed identification scheme for GPAC}

Many strains of GPAC grow so slowly, even on enriched blood agar, that their identification is of little clinical relevance. Therefore, we propose the following rapid identification scheme, which in most cases should be completed within $48 \mathrm{~h}$ of isolation. Any of the tests used in this study can be incorporated with slight modifications.

1. A colony from a primary isolation plate is examined by microscopy (gram-stained smear) and inoculated to give a heavy growth over half an enriched blood agar plate, then streaked to produce individual colonies on that plate. A metronidazole 5- $\mu \mathrm{g}$ disk is placed on the edge of the plate at the edge of the zone of heavy growth and the plate is incubated anaerobically. A subculture of the colony is also incubated in air $+\mathrm{CO}_{2} 6 \%$ to detect capnophilic organisms.

2. After $48 \mathrm{~h}$, the presence of a zone around the metronidazole disk is checked. Also, there should be no visible growth on the aerobic plate. The gram-stained smear should be repeated, preferably from an isolated colony, if the original was from a colony more than $48 \mathrm{~h}$ old. A pre-formed enzyme kit is inoculated from the area of heavy growth, and also a purity plate. The colonial morphology (size, shape and colour of colonies) is noted. The original plate is then re-incubated with the purity plate.

The enzyme kit is read after incubation for $4 \mathrm{~h}$; the majority of strains are likely to be identified as $P$. magnus, $P$. micros, $P$. anaerobius or $P$. asaccharolyticus. Strains which are not identified by this stage can be placed in a VFA group by extracting VFAs from the agar with ether, followed by GLC of the extract. $^{18}$

3. The colonial morphology after incubation for 5 days can, with experience, be of assistance for some strains of the butyric group, e.g., P. asaccharolyticus group B.

The following modifications may be appropriate:

1. Many strains of GPAC are slow-growing; if their colonies are smaller than $1 \mathrm{~mm}$ diameter after incubation for $48 \mathrm{~h}$, two plates should be inoculated to harvest an adequate growth for the enzyme kit.

2. A pleomorphic coccobacillus arranged in chains, with appropriate colonies smelling of plasticine, 
can be identified as a presumptive strain of $P$. anaerobius. A plate should be inoculated as above, and an SPS disk (as well as a metronidazole disk) placed in the area of heavy growth. The disk-test results can be read usually after $24 \mathrm{~h}$. If the strain is sensitive to SPS, a definitive identification of $P$. anaerobius can be made; there is no need to inoculate an enzyme kit.

3. If a small coccus is seen on the gram-stained film, the application of a novobiocin disk to the area of primary inoculum may help to separate strains of $P$. micros (resistant) from $P$. heliotrinreducens (sensitive).

4. If the microscopic appearance from the primary plate suggests a very decolourised strain of $P$.

\section{References}

1. Watt B, Jack EP. What are anaerobic cocci? J Med Microbiol $1977 ; 10: 461-468$

2. Brook I. Recovery of anaerobic bacteria from clinical specimens in 12 years at two military hospitals. J Clin Microbiol 1988; 26: 1181-1188

3. Gorbach SL. Anaerobic cocci. In: Mandell GL, Douglas RG, Bennett JE (eds) Principles and practice of infectious diseases, 2nd edn. New York, John Wiley. 1985: 13731375.

4. Wren MWD, Baldwin AWF, Eldon CP, Sanderson PJ. The anaerobic culture of clinical specimens: a 14-month study. J Med Microbiol 1977; 10: 49-61

5. Holdeman LV, Cato EP, Moore WEC. Anaerobe laboratory manual, 4th edn. Blacksburg, Virginia Polytechnic Institute and State Univerity. 1977.

6. Sutter VL, Citron DM, Edelstein MAC, Finegold SM Wadsworth anaerobic bacteriology manual, 4th edn. Lo Angeles, Star Publishing Co. 1985.

7. Parker MT. The anaerobic gram-positive cocci. In: Wilson G et al. (eds) Topley and Wilson's principles of bacteriology, virology and immunity, 7th edn, vol 2. London, Edward Arnold. 1983: 238-239.

8. Holdeman Moore LV, Johnson JL, Moore WEC Genus Peptococcus and Genus Peptostreptococcus. In: Sneath PHA (ed) Bergey's Manual of systematic bacteriology, vol 2. Baltimore, Williams and Wilkins. 1986: 1082-1092.

9. Ezaki T, Yamamoto N, Ninomiya K, Suzuki S, Yabuuchi E. Transfer of Peptococcus indolicus, Peptococcus asaccharolyticus, Peptococcus prevotii and Peptococcus magnus to the genus Peptostreptococcus and proposal of Peptostreptococcus tetradius sp. nov. Int J Syst Bact 1983; 33: 683-698.

10. Huss VAR, Festl H, Schleifer KH. Nucleic acid hybridization asaccharolyticus, a vancomycin disk placed on the area of heavy growth can be used to exclude gramnegative cocci.

D.A.M. is very grateful to the Sir Jules Thorn Charitable Trust for the financial support which made this study possible. We are grateful to Professor S. Tabaqchali for her support, and to the staff of the Department of Medical Microbiology, St Bartholomew's Hospital, for isolating and saving many of these strains. In particular, M. Wilks gave much of his time to run the GLC and helped with advice about this paper; Shirley Lawrence and A. Koh assisted in the laboratory. Dr J. M. Hardie (The London Hospital Dental School), Dr B. Watt (City Hospital, Edinburgh) and Professor I. Phillips (St Thomas' Hospital) kindly supplied us with reference strains. Professor B. I. Duerden (University of Sheffield Medical School) and D. Quinn (St Thomas' Hospital) commented on early drafts of this paper. Dr B. Thomas of API-BioMerieux gave us much advice about the enzyme kits.

studies and deoxyribonucleic acid base compositions of anaerobic, gram-positive cocci. Int J Syst Bact 1984; 34: 95-101.

11. Ezaki T, Yabuuchi E. Oligopeptidase activity of gram-positive anaerobic cocci used for rapid identification. J Gen Appl Microbiol $1985 ; 31$ : 255-265.

12. Murdoch DA, Mitchelmore IJ, Tabaqchali S. Identification of gram-positive anaerobic cocci by use of systems for detecting pre-formed enzymes. $J$ Med Microbiol 1988; 25 : 289-293.

13. Murdoch DA, Mitchelmore IJ, Nash RA, Hardie JM, Tabaqchali S. Pre-formed enzyme profiles of reference strains of gram-positive anaerobic cocci. J Med Microbiol 1988; 27 : 65-70.

14. Wren MWD, Eldon CP, Dalin GH. Novobiocin and the differentiation of peptococci and peptostreptococci. $J$ Clin Pathol 1977; 30: 620-622.

15. Cato EP, Johnson JL, Hash DE, Holdeman LV. Synonomy of Peptococcus glycinophilus (Cardon and Barker 1946) Douglas 1957 with Peptostreptococcus micros (Prévot 1933) Smith 1957 and electrophoretic differentiation of Peptostreptococcus micros from Peptococcus magnus (Prévot 1933) Holdeman and Moore 1972. Inst J Syst Bact 1983; 33: 207-210.

16. Harpold DJ, Wasilauskus BL. Rapid identification of obligately anaerobic gram-positive cocci using high-performance liquid chromatography. J Clin Microbiol 1987; 25: 9961001

17. Holdeman LV, Moore WEC. New genus, Coprococcus, twelve new species, and emended descriptions of four previously described species of bacteria from human feces. Int $J$ Syst Bact 1974; $24: 260-277$.

18. Wiggins RJ, Wilks M, Tabaqchali S. Analysis by gas liquid chromatography of production of volatile fatty acids by anaerobic bacteria grown on solid medium. J Clin Pathol $1985 ; 38: 933-936$ 